\title{
Impact of Fano and Breit-Wigner resonances in the thermoelectric properties of nanoscale junctions
}

\author{
V. M. García-Suárez, ${ }^{1,2,3}$ R. Ferradás, ${ }^{1,2}$ and J. Ferrer ${ }^{1,2,3}$ \\ ${ }^{1}$ Departamento de Física, Universidad de Oviedo, 33007 Oviedo, Spain \\ ${ }^{2}$ Nanomaterials and Nanotechnology Research Center (CINN), Oviedo, Spain \\ ${ }^{3}$ Department of Physics, Lancaster University, Lancaster LAI 4YB, United Kingdom
}

(Received 10 July 2013; revised manuscript received 22 October 2013; published 16 December 2013)

\begin{abstract}
We show that the thermoelectric properties of nanoscale junctions featuring states near the Fermi level strongly depend on the type of resonance generated by such states, which can be either Fano- or Breit-Wigner-like. We give general expressions for the thermoelectric coefficients generated by the two types of resonances and calculate the thermoelectric properties of these systems, which encompass most nanoelectronics junctions. We include simulations of real junctions where metalloporphyrins dithiolate molecules bridge gold electrodes and prove that for some metallic elements the thermoelectric properties show a large variability with respect to the position of the resonance near the Fermi level. We find that the thermopower and figure of merit are largely enhanced when the resonance gets close to the Fermi level and reach values higher than typical values found in other nanoscale junctions. The specific value and temperature dependence are determined by a series of factors such as the strength of the coupling between the state and other molecular states, the symmetry of the state, the strength of the coupling between the molecule and the leads and the spin-filtering behavior of the junction.
\end{abstract}

DOI: 10.1103/PhysRevB.88.235417

PACS number(s): 72.20.Pa, 73.63.-b, 85.35.Ds, 71.15.-m

\section{INTRODUCTION}

The use of molecules as electronic components is expected to surpass, at least temporally, one of the limits imposed on Moore's law as the size of the electronic components shrinks towards the atomic limit. Their particular properties would allow us to develop more involved and efficient circuits and electronic devices with sizes much smaller than conventional silicon-based devices. ${ }^{1}$ Among the properties that molecules in metallic junctions can show are rectification, ${ }^{2}$ negative differential resistance, ${ }^{3}$ switching, ${ }^{4}$ memory, ${ }^{5}$ and sensing. ${ }^{6}$ Adding a thermal gradient or a coupling to a thermal bath ${ }^{7,8}$ would also allow molecules to work as nanometer-size thermoelectric devices, ${ }^{9}$ which could be used in applications ranging from chip cooling to building refrigerators. One of the thermoelectric coefficients, the Seebeck coefficient $S$, is also especially suited to gather information on the mechanisms of molecular conduction ${ }^{10}$ and the chemistry of the junction. ${ }^{11}$ For instance, from the sign of $S$ it is possible to deduce if the Fermi level lies close to the highest occupied molecular orbital (HOMO) or the lowest unoccupied molecular orbital (LUMO). ${ }^{12,13}$ A positive (negative) sign indicates $p$-type ( $n$-type) conduction, which means the Fermi level lies near the HOMO (LUMO). This implies that the sign and magnitude of the thermopower can be changed by gating the molecule. ${ }^{14-16}$ The Seebeck coefficient is also a very sensible magnitude that depends on factors such as the molecular length, ${ }^{17,18}$ the molecular conformation, ${ }^{19}$ the contact group ${ }^{20}$ the side groups, ${ }^{21-23}$ the surface reconstruction, ${ }^{24}$ and the type of electrodes. ${ }^{25}$

The most important quantity that measures the thermoelectric efficiency of a system is the dimensionless figure of merit $Z T=S^{2} G T / \kappa$, which is proportional to the square of the Seebeck coefficient $S$ and the conductance $G$ and inversely proportional to the thermal conductance $\kappa$. This number, which determines how easy it is to transform heat into electricity, should be as high as possible (closer to 1 or higher) in order for a thermoelectric device to work effectively. Values larger than $1(\sim 2.4)$ have already been measured in inorganic superlattice devices. ${ }^{26}$ In the field of molecular electronics, however, despite current efforts, ${ }^{11,27-32}$ the measured $S$ and $Z T$ are not yet very high $[|S| \sim 33 \mu \mathrm{V} / \mathrm{K}$ (Ref. 30) and $Z T \sim 0.25$ (Ref. 31)]. Theoretical calculations predict that much higher values should be achieved when Fano resonances ${ }^{21,33}$ or interference-related peaks ${ }^{34}$ cross the Fermi level, but such predictions have not been confirmed experimentally yet. These values, calculated in the framework of coherent transport, should also be corrected by taking into account the phonon thermal conductance, ${ }^{35}$ inelastic scattering, ${ }^{36}$ and, in general, coupling to phonons. ${ }^{37}$

In this paper we calculate the thermoelectric coefficients and figure of merit of molecules which show interferencerelated features around the Fermi level ${ }^{38}$ in the form of BreitWigner-like and Fano resonances. In Sec. II we give a brief theoretical introduction on the thermoelectric coefficients. In Sec. III, we include $a b$ initio simulations of molecular wires (metalloporphyrins dithiolate). Finally, in Sec. IV we present a model that can be used to study the evolution of the thermoelectric properties of molecular wires as a function of a series of parameters, among which are the type of resonance that crosses the Fermi level, which is related to the symmetry of the state, the strength of coupling between the resonance and other molecular levels, and the coupling between the molecule and the electrodes.

\section{THERMOELECTRIC COEFFICIENTS}

When a junction is subject to an electrostatic potential difference and a temperature gradient, electric and heat current flow from one electrode to another. With just an electrostatic potential, electrons move from the negative to the positive electrode, whereas the electric current is defined to flow in the opposite direction. With a temperature gradient, however, there is no rule of thumb that allows one to determine how the current flows without accurate information on the electronic structure, 
i.e., the transmission, of the junction. If the transmission below the Fermi level is higher, electrons flow from the cold to the hot electrode, whereas the contrary happens if the transmission is higher above the Fermi level. The electrostatic potential and the temperature gradient generate also a heat current which, in general, flows from the hot to the cold electrode. For a system with spin polarization, ${ }^{39,40}$ this is summarized in the following equation:

$$
\left(\begin{array}{c}
I \\
\dot{Q}
\end{array}\right)=\frac{1}{h}\left(\begin{array}{cc}
e^{2} L_{0}^{\mathrm{t}} & \frac{e}{T} L_{1}^{\mathrm{t}} \\
e L_{1}^{\mathrm{t}} & \frac{1}{T} L_{2}^{\mathrm{t}}
\end{array}\right)\left(\begin{array}{c}
\Delta V \\
\Delta T
\end{array}\right),
$$

where $I$ and $\dot{Q}$ are the electric and heat currents, respectively, and the moments $L_{n}^{\mathrm{t}}=L_{n}^{\uparrow}+L_{n}^{\downarrow}(n=0,1,2)$ of the transmission coefficients are given by ${ }^{41}$

$$
L_{n}^{\sigma}=\int_{-\infty}^{\infty}\left(E-E_{\mathrm{F}}\right)^{n} T^{\sigma}(E) \frac{\partial f(E, V, T)}{\partial E} d E,
$$

where $f$ is the Fermi distribution function, which depends on voltage and temperature. Equation (1) can be expressed in terms of measurable thermoelectric quantities: the electric conductance $G$, thermopower $S$, Peltier coefficient $\Pi$, and the electronic contribution to the thermal conductance $\kappa$ :

$$
\left(\begin{array}{c}
\Delta V \\
\dot{Q}
\end{array}\right)=\left(\begin{array}{ll}
R & S \\
\Pi & \kappa
\end{array}\right)\left(\begin{array}{c}
I \\
\Delta T
\end{array}\right),
$$

where

$$
\begin{gathered}
G=\frac{e^{2}}{h} L_{0}^{\mathrm{t}}, \\
S=-\frac{1}{e T} \frac{L_{1}^{\mathrm{t}}}{L_{0}^{\mathrm{t}}}, \\
\Pi=\frac{1}{e} \frac{L_{1}^{\mathrm{t}}}{L_{0}^{\mathrm{t}},} \\
\kappa=\frac{1}{h T}\left(L_{2}^{\mathrm{t}}-\frac{L_{1}^{\mathrm{t} 2}}{L_{0}^{\mathrm{t}}}\right) .
\end{gathered}
$$

Notice that according to these formulas, in order to produce the highest thermopower it is necessary to have the factor in the denominator $\left(L_{0}^{\mathrm{t}}=L_{0}^{\uparrow}+L_{0}^{\downarrow}\right)$ be as small as possible. However, as we will see later, in a system with spin polarization both channels are usually different around the Fermi level, one of them being much larger than the other in some cases, which decreases the value of $S$.

The figure of merit can also be expressed in terms of the transmission moments by substituting the above expressions in the $Z T$ definition:

$$
Z T=\frac{1}{\frac{L_{0}^{\mathrm{t}} L_{2}^{\mathrm{t}}}{L_{1}^{\mathrm{t} 2}}-1} .
$$

In this case the figure of merit becomes large when the factor of the moments in the denominator decreases towards 1 .

Approximations to these expressions can be obtained in the limit of low temperatures by expanding $T(E)$ about $E=E_{\mathrm{F}}$, which we take to be equal to $0 \mathrm{eV}$. In the case of a single level coupled to featureless leads, whose transmission is given by a
Breit-Wigner resonance,

$$
T(E)=\frac{\Gamma^{2}}{\left(E-\epsilon_{0}\right)^{2}+\Gamma^{2}},
$$

where $\Gamma$ is the strength of the coupling of the level to the leads and $\epsilon_{0}$ is the energy of the level, the low- $T$ bias conductance, Seebeck coefficient, and figure of merit are given by

$$
\begin{gathered}
G=G_{0} \frac{\Gamma^{2}}{\epsilon_{0}^{2}+\Gamma^{2}}, \\
S=-G_{\text {th }} \frac{2 h}{e} \frac{\epsilon_{0}}{\epsilon_{0}^{2}+\Gamma^{2}}, \\
Z T=\frac{4 \mathrm{~S}_{0} \epsilon_{0}^{2}}{\left(\epsilon_{0}^{2}+\Gamma^{2}\right)^{2}-4 \mathrm{~S}_{0} \epsilon_{0}^{2}},
\end{gathered}
$$

where $G_{0}=2 e^{2} / h$ and $G_{\mathrm{th}}=\pi^{2} k_{\mathrm{B}}^{2} T / 3 h$ are the electrical and thermal conductance quantum units and $S_{0}=h T G_{\text {th }}$.

For a Fano resonance produced by a side level with the same on-site energy as the backbone level $\left(\epsilon_{0}\right)$ and coupled to the latter by a matrix element $V$, the transmission is

$$
T(E)=\frac{\Gamma^{2}\left(E-\epsilon_{0}\right)^{2}}{\left[\left(E-\epsilon_{0}\right)^{2}-V^{2}\right]^{2}+\Gamma^{2}\left(E-\epsilon_{0}\right)^{2}},
$$

and the thermoelectric coefficients $G$ and $S$ and figure of merit are given by

$$
\begin{gathered}
G=G_{0} \frac{\Gamma^{2} \epsilon_{0}^{2}}{\left(\epsilon_{0}^{2}-V^{2}\right)^{2}+\Gamma^{2} \epsilon_{0}^{2}}, \\
S=-G_{\text {th }} \frac{2 h}{e} \frac{\Delta(0)}{\epsilon_{0} \Gamma^{2}}, \\
Z T=\frac{\mathrm{S}_{0} \Delta^{2}(0)}{\epsilon_{0}^{2} \Gamma^{4}-\mathrm{S}_{0} \Delta^{2}(0)},
\end{gathered}
$$

where $\Delta(0)=\left[\left(2 \epsilon_{0}^{2}-2 V^{2}+\Gamma^{2}\right) T(0)-\Gamma^{2}\right]$. Notice the Fano resonance diverges at $\epsilon_{0}=0$, which is an unphysical singularity. This can be avoided by including a background transmission due to other resonances, which are always present in realistic systems.

With these expressions it is then possible to calculate the thermoelectric coefficients in a given junction, provided the transmission is known. In general, in order to obtain large $S$ and $Z T$ it is convenient to have large derivatives around the Fermi level since the first moment $\left(L_{1}\right)$ is, at least at low temperatures, proportional to the derivative of the transmission. Notice again that these expressions can only be used at low temperatures, but qualitative trends derived from them (i.e., the overall shape of the thermoelectric coefficients as a function of the level position) are still valid at large temperatures.

\section{FIRST-PRINCIPLES CALCULATIONS}

Molecular junctions that show sharp features around the Fermi level could be very good candidates to act as thermoelectric enhancers. In particular, molecular junctions based on metalloporphyrin wires, which have recently been the subject of a 
lot of interest, both theoretically ${ }^{42-47}$ and experimentally, ${ }^{48,49}$ show, for certain metallic elements, a series of resonances close to the Fermi level which can be Breit-Wigner- or Fano-like. This property makes them especially appealing for thermoelectricity since such resonances can be employed to finely tune the thermoelectric response with a gate voltage (see below).

In order to have a clear picture of the influence of the electronic properties on the thermoelectric response we initially calculated from first principles the electronic and transport properties of metalloporphyrins dithiolate molecules between gold electrodes. In the next section we pay attention to the most important features of these systems with the help of a simple model. The metallic elements which produce states close to the Fermi level are $\mathrm{Fe}$ and $\mathrm{Cu}$ atoms. ${ }^{47}$ We therefore focus in this study on only $\mathrm{Fe}$ and $\mathrm{Cu}$ metalloporphyrins dithiolate.

The first-principles calculations were performed in the framework of density functional theory (DFT). ${ }^{50}$ We used the SIESTA code, ${ }^{51}$ which employs norm-conserving pseudopotentials and a basis set of pseudoatomic orbitals. We included nonlinear core corrections ${ }^{52}$ in the transition-metal pseudopotentials to correctly account for the overlap between the valence and the core states. We used for gold a single- $\zeta$ basis (SZ) with explicit $s$ and $d$ orbitals as valence orbitals. For all the other elements ( $\mathrm{H}, \mathrm{C}, \mathrm{O}, \mathrm{N}, \mathrm{S}$, and transition-metal) we used a double- $\zeta$ polarized basis (DZP). The exchange and correlation potential was approximated with the generalized gradient approximation (GGA), as parameterized by Perdew, Burke, and Ernzerhof. ${ }^{53}$ We defined the real-space grid with an energy cutoff of $400 \mathrm{Ry}$. We performed the structural relaxations and transport calculations in the $\Gamma$ point, which was enough to relax the coordinates and correctly determine the transmission around the Fermi level. We also did tests with $k$ points $(2 \times 2$ in the perpendicular directions), and the results were essentially the same around the Fermi level. We relaxed the coordinates until all forces were smaller than $0.01 \mathrm{eV} / \AA$.

We corrected the self-interaction and other errors produced by DFT by using the DFT $+U$ approach, which yields qualitatively correct results in systems with transition-metal atoms. ${ }^{54}$ Adding this parameter to the central metallic atom was equivalent to adding a gate potential which moves the states associated with it, as we shall see. Take into account, however, that without a gate voltage only the results with $U$ can be trusted. To reproduce previous theoretical results for the gas-phase iron metalloporphyrin, ${ }^{55,56}$ we used $U=4.5 \mathrm{eV}$. This value was also employed in the molecule between electrodes and in other metallic elements. Notice that small differences in the $U$ term due to the electrodes or other metallic atoms do not affect the results and the only effect is a slight movement of the resonances around the Fermi level, which produces qualitatively similar results.

Structurally, the gold electrodes were grown in the (001) direction. The sulfur atoms were contacted to the gold surfaces in the hollow position, which was found to be more stable than the top and bottom configurations, at a distance of $1.8 \AA$. The transport calculations were performed with the SMEAGOL and GOLLUM codes. ${ }^{57,58}$ According to the transport formalism, the junction was divided in three parts: the left and right leads and extended molecule (EM), which included the central part of
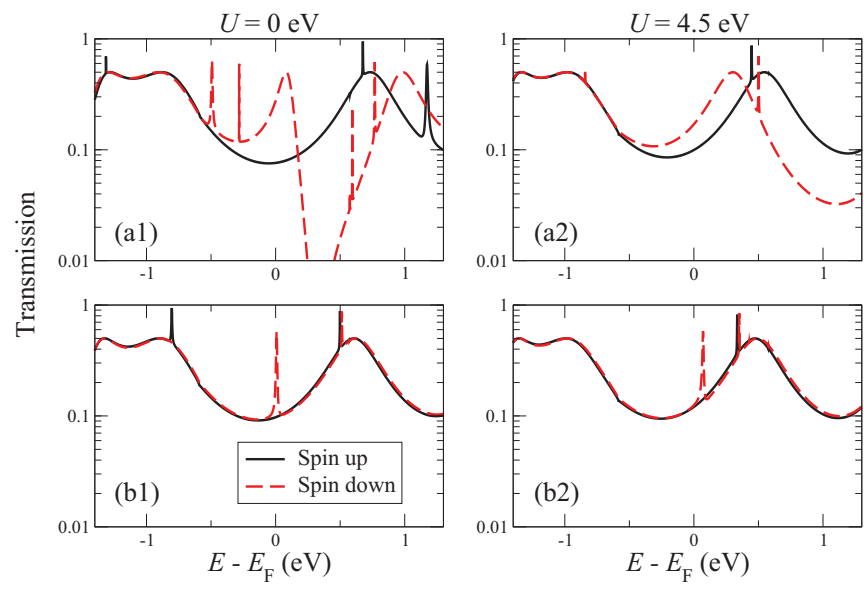

FIG. 1. (Color online) Transmission coefficients for (a) Fe and (b) $\mathrm{Cu}$ metalloporphyrins dithiolate between gold electrodes, calculated with GGA and $U=0 \mathrm{eV}$ (left) and $U=4.5 \mathrm{eV}$ (right).

the junction and also some layers of the gold leads to make sure that the electronic structure was converged to the bulk electronic structure away from the surfaces.

We show in Fig. 1 the transmission of $\mathrm{Fe}$ and $\mathrm{Cu}$ metalloporphyrins dithiolate between gold electrodes, with and without the $U$ correction. As can be seen, the bare Fe case shows a very clear Fano resonance with its antiresonance close to the Fermi level. When the $U$ term is added, the resonance moves to higher energies, and its effect on the transmission around the Fermi level decreases. In the $\mathrm{Cu}$ case, however, there seems to be a sharp Breit-Wigner resonance which also moves to higher energies when the $U$ term is included.

From the transmissions we calculate the thermoelectric properties by using the equations in Sec. II. The results are shown in Figs. 2 and 3. The temperature dependence on the horizontal axis enters in the Fermi distribution function, as explained before. In the case of iron the electric conductance
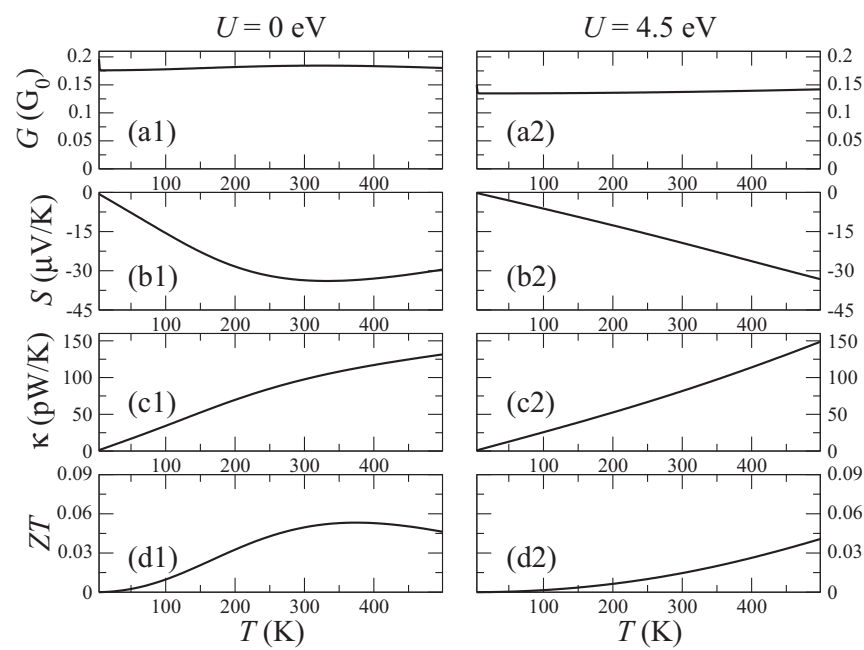

FIG. 2. Thermoelectric coefficients for Fe metalloporphyrins dithiolate between gold electrodes, calculated with GGA and $U=$ $0 \mathrm{eV}$ (left) and $U=4.5 \mathrm{eV}$ (right): (a) conductance $G$, (b) Seebeck coefficient $S$, (c) thermal conductance $\kappa$, and (d) figure of merit $Z T$. 

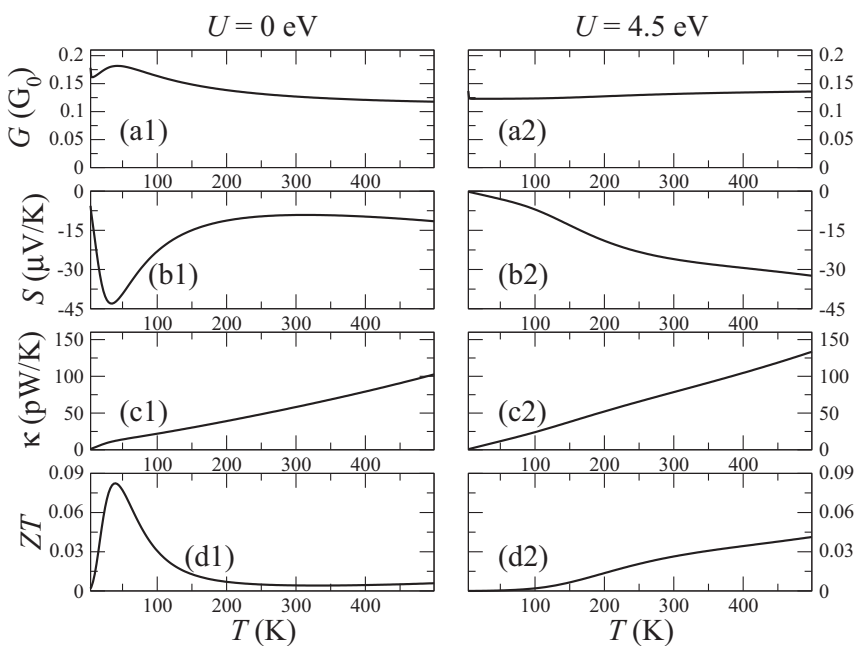

FIG. 3. Thermoelectric coefficients for $\mathrm{Cu}$ metalloporphyrins dithiolate between gold electrodes, calculated with GGA and $U=$ $0 \mathrm{eV}$ (left) and $U=4.5 \mathrm{eV}$ (right): (a) conductance $G$, (b) Seebeck coefficient $S$, (c) thermal conductance $\kappa$, and (d) figure of merit $Z T$.

is almost constant, whereas the thermal conductance increases roughly linearly with temperature. The Seebeck coefficient, which is negative and signals that the Fermi level is close to the LUMO, is relatively large, and its evolution with temperature qualitatively changes when the $U$ term is included and the state moves to higher energies. The same happens to the figure of merit, but it is rather small. These evolutions can be explained by taking into account the spin-polarized Fano resonance, which is a bit above the Fermi level and produces large changes in the derivative of the transmission $\left(L_{1}\right)$ without $U$ but move to higher energies when the $U$ term is included and therefore leaves a smoother transmission at the Fermi level.

The copper case is more interesting, as the temperature evolution of some quantities has more features and changes more dramatically when the state moves. Again, the electric conductance is rather constant, and the thermal conductance increases linearly. The Seebeck coefficient shows, however, a dip at low temperatures and becomes almost constant as the temperature increases. The dip disappears when the state moves to higher energies and the magnitude decreases to more negative values with $T$. The figure of merit is again small but has a peak at low temperatures. Such a peak disappears when the $U$ term is included and is substituted by a smooth increase. This evolution is a consequence of the presence at the Fermi level of a sharp spin-polarized resonance which moves closer to the LUMO when the $U$ term is included. Such resonance, which is just a bit above the Fermi level, gives rise to large derivatives and therefore dramatically increases, in absolute value, the Seebeck coefficient $S$. This coefficient is, however, not very large because the sum of the transmission $\left(L_{0}\right)$ of both spin channels is not small.

The copper molecule could be a candidate to show large thermoelectric properties due to the presence of a resonance close to the Fermi level. However, the introduction of $U$ moves the resonance to higher energies and decreases the thermoelectric response. It is then interesting to consider the case of applying a gate voltage that brings back the resonance
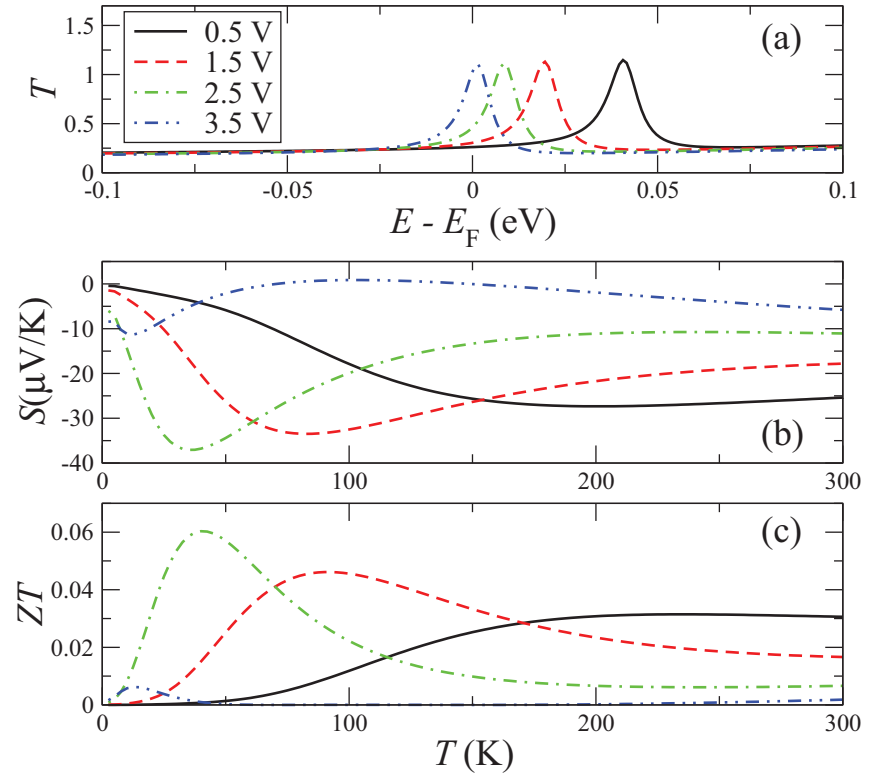

FIG. 4. (Color online) (a) Spin-polarized transmission as a function of energy and (b) Seebeck coefficient and (c) figure of merit as a function of temperature for $\mathrm{Cu}$ metalloporphyrins dithiolate between gold electrodes, calculated with GGA, $U=4.5 \mathrm{eV}$, and different gate potentials.

to the Fermi level. We show that in Fig. 4. As can be seen, moving the resonance to lower energies increases the absolute value of both $S$ and $Z T{ }^{59}$ The highest values are obtained when the largest slope of the resonance is just at the Fermi
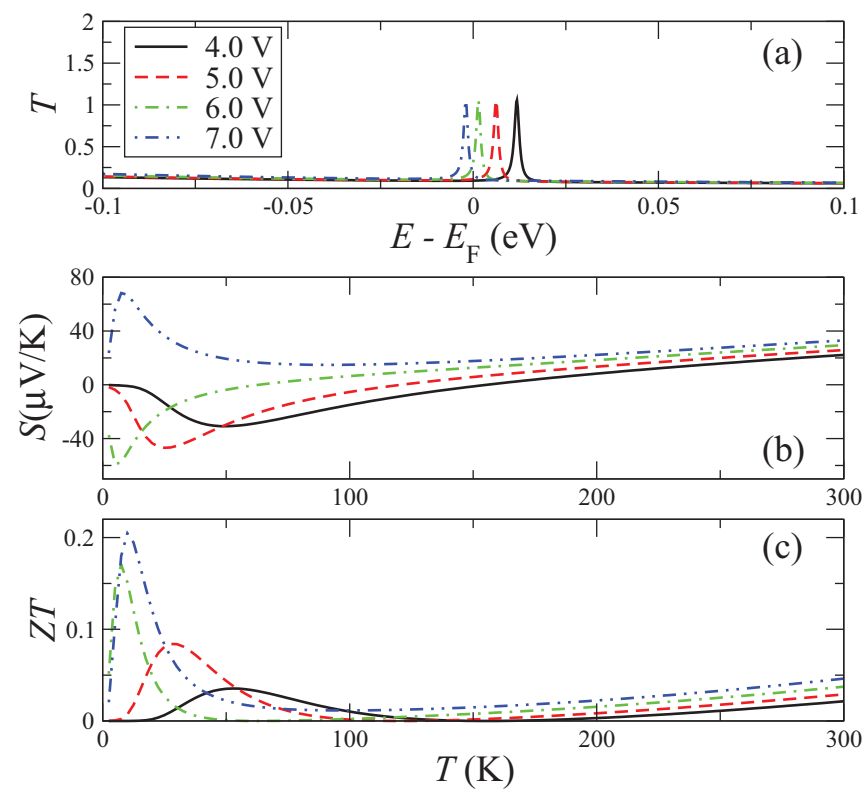

FIG. 5. (Color online) (a) Spin-polarized transmission as a function of energy and (b) Seebeck coefficient and (c) figure of merit as a function of temperature for stretched $\mathrm{Cu}$ metalloporphyrins dithiolate between gold electrodes (the contact distance between the molecule and the electrodes was increased $0.8 \AA$ on each side of the equilibrium configuration), calculated with GGA, $U=4.5 \mathrm{eV}$, and different gate potentials. 

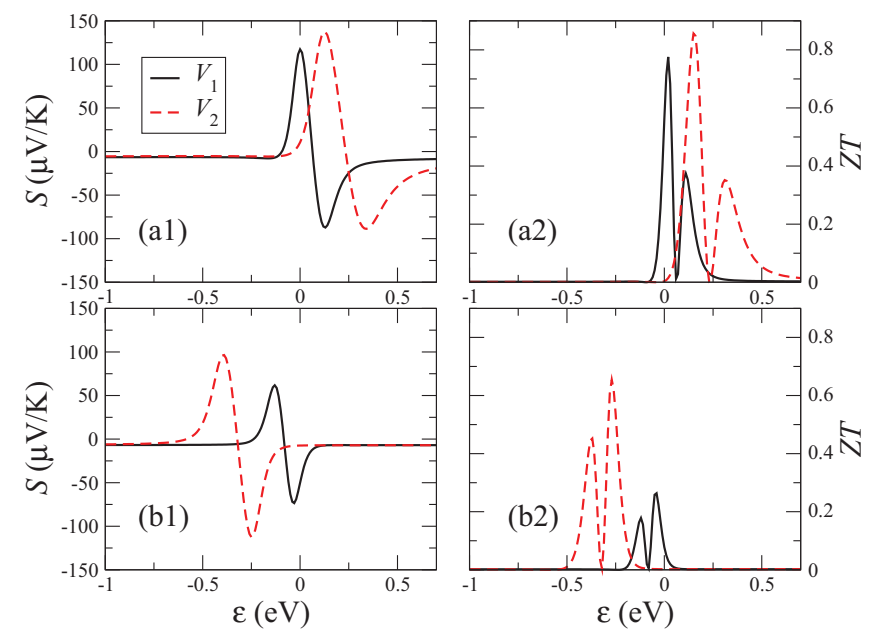

FIG. 6. (Color online) Seebeck coefficient $S$ (left) and figure of merit $Z T$ (right) for a model system with (a) a Fano and (b) a BreitWigner-like resonance in the HOMO-LUMO gap, calculated at $T=$ $250 \mathrm{~K}$. In the case of the Fano (Breit-Wigner-like) resonance the coupling between the $\pi(\sigma)$ level and the $d$ level is $V_{1}=0.2 \mathrm{eV}$ $\left(V_{2}=0.4 \mathrm{eV}\right)$, which corresponds to the solid (dashed) lines.

level $\left(V_{\mathrm{G}}=2.5 \mathrm{~V}\right)$. Beyond that point $S$ and $Z T$ decrease $\left(V_{\mathrm{G}}=3.5 \mathrm{~V}\right)$. The increase of the thermoelectric properties is not very spectacular, however, due to the fact that the resonance is not extremely sharp. More acute resonances can

$\varepsilon$

(a)

(a) $\begin{array}{rrrrrrrrrr}-1 & -0.8 & -0.6 & -0.4 & -0.2 & 0 & 0.2 & 0.4 & 0.6 & 0.8\end{array}$

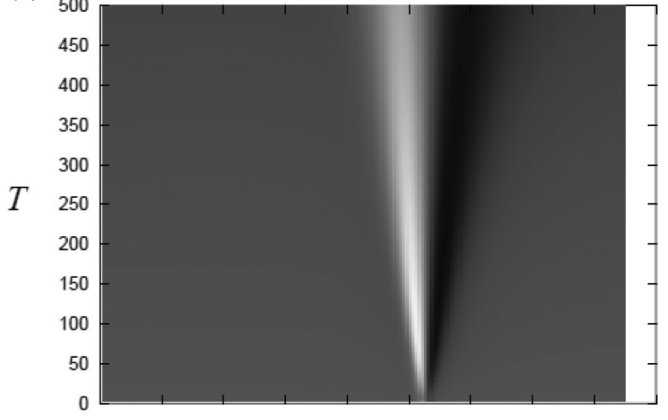

$\varepsilon$

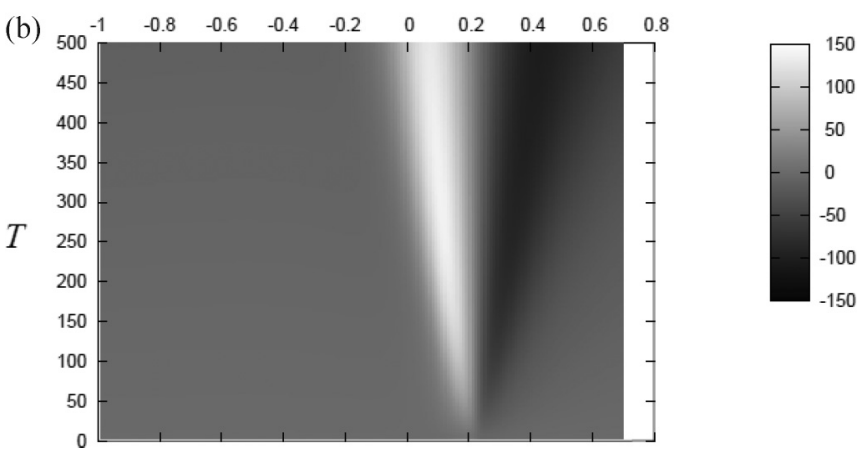

FIG. 7. Seebeck coefficient $S$ in units of $\mu \mathrm{V} / \mathrm{K}$ for model metalloporphyrins dithiolate which have a Fano-like resonance around the Fermi level as a function of temperature $T$ (in K) and the level position $\epsilon_{0}$ (in $\mathrm{eV}$ ). The coupling between the $d$ state and the $\pi$ state is (a) 0.2 and (b) $0.4 \mathrm{eV}$. be, however, obtained by decreasing the coupling between the molecule and the gold electrodes, which reduces the width of all transmission features. We show in Fig. 5 results for a system where the distance between the gold electrodes and the sulfur atoms on each side increases by $0.8 \AA$ relative to the equilibrium configuration. As can be seen, now the resonance is very sharp, and the changes in the thermoelectric properties are more spectacular. Notice that the thermopower changes sign when the resonance crosses the Fermi level due to the change of slope. Based on these results, we can claim that stretching molecular electronic junctions increases, in general, the thermoelectric performance.

\section{TIGHT-BINDING MODEL}

\section{A. General properties}

The most important features in the transmission of metalloporphins dithiolate junctions can be reproduced with a simple model. With such a model we have analyzed in detail the impact of Fano resonances on the charge transport properties of these systems. ${ }^{47} \mathrm{We}$ have found that these molecules contribute with three broad resonances to the transmission coefficients of the junctions, which correspond to HOMO-1 (a $\sigma$ molecular orbital), the HOMO, and the LUMO (which has $\pi$ character). In addition, the $3 d$ atom contributes with a spin-polarized strongly localized state, which hybridizes with HOMO- 1 or the LUMO, depending on the element, and gives rise to a Fano resonance. To model these junctions, we use the
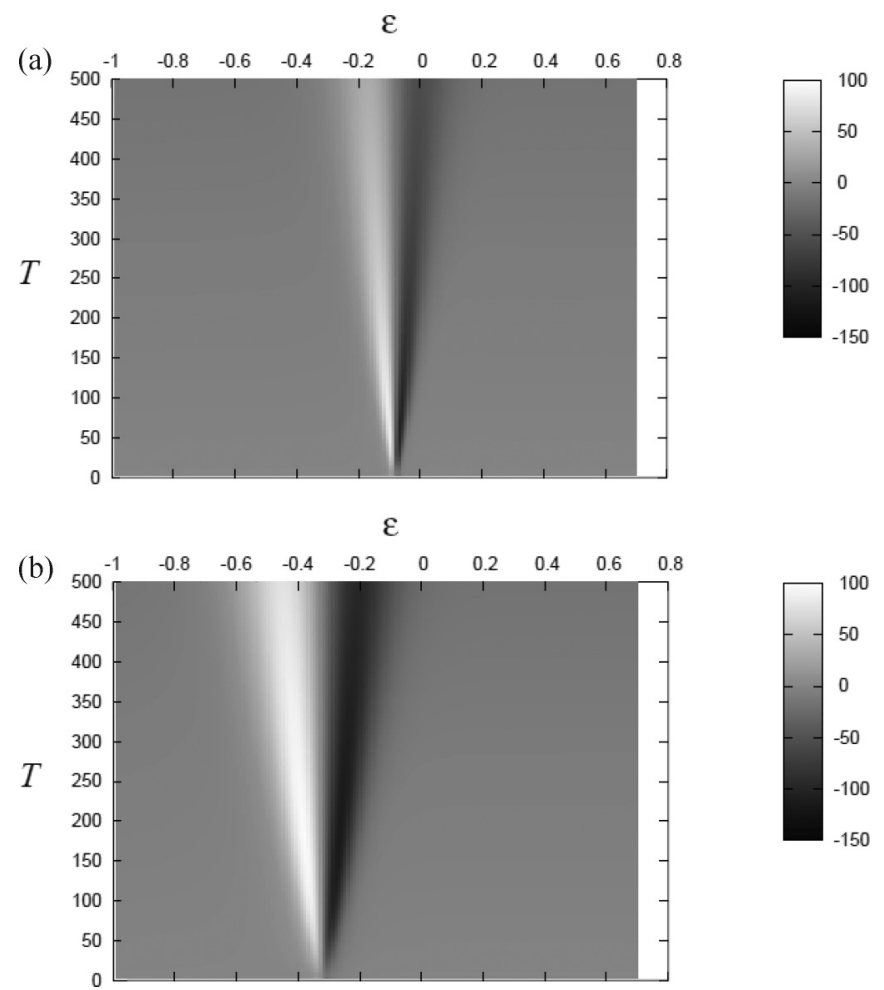

FIG. 8. Seebeck coefficient $S$ in units of $\mu \mathrm{V} / \mathrm{K}$ for model metalloporphyrins dithiolate which have a Breit-Wigner-like resonance around the Fermi level as a function of temperature $T$ (in $\mathrm{K}$ ) and the level position $\epsilon_{0}$ (in eV). The coupling between the $d$ state and the $\sigma$ state is (a) 0.4 and (b) $0.8 \mathrm{eV}$. 


\section{Hamiltonian}

$$
\begin{gathered}
\hat{\mathcal{H}}=\hat{\mathcal{H}}_{\text {gold }}+\hat{\mathcal{H}}_{\mathrm{M}}+\hat{\mathcal{H}}_{\text {gold }-\mathrm{M}}, \\
\hat{\mathcal{H}}_{\text {gold }}=\sum_{k, \sigma} \epsilon_{k} c_{k \sigma}^{\dagger} c_{k \sigma}, \\
\hat{\mathcal{H}}_{\mathrm{M}}=\sum_{\sigma} \epsilon_{d \sigma} \hat{d}_{\sigma}^{\dagger} \hat{d}_{\sigma}+\sum_{i=1,2,3, \sigma} \epsilon_{i} \hat{c}_{i \sigma}^{\dagger} \hat{c}_{i \sigma} \\
+\sum_{i=1,3} V_{i}\left(\hat{c}_{i \sigma}^{\dagger} \hat{d}_{\sigma}+\hat{d}_{\sigma}^{\dagger} \hat{c}_{i \sigma}\right), \\
\hat{\mathcal{H}}_{\text {gold-M }}=\sum_{k, i, \sigma} T_{i}\left(c_{k \sigma}^{\dagger} c_{i \sigma}+c_{i \sigma}^{\dagger} c_{k \sigma}\right),
\end{gathered}
$$

where the operators $\hat{c}_{i, \sigma}, i=1,2,3$, represent the HOMO-1, HOMO, and LUMO molecular levels. The operator $\hat{d}$ represents the $d$ level associated with the central metallic atom. We assume that this level couples only to either the HOMO-1 or the LUMO levels (e.g., only $V_{1}$ or $V_{3}$ is different from zero). We assume a wide-band approximation for the band structure of the gold electrodes, so that its density of states $\rho_{e}$ and therefore the Gamma matrices $\left(\Gamma_{i}=T_{i}^{2} \rho_{e}\right)$ are constant. Finally, we assume that the $d$ level is spin polarized so that only the spin-up $d$ level enters the relevant energy window.

By using this model we have found ${ }^{47}$ that the presence of two types of resonances can be explained by how the $d$

(a)
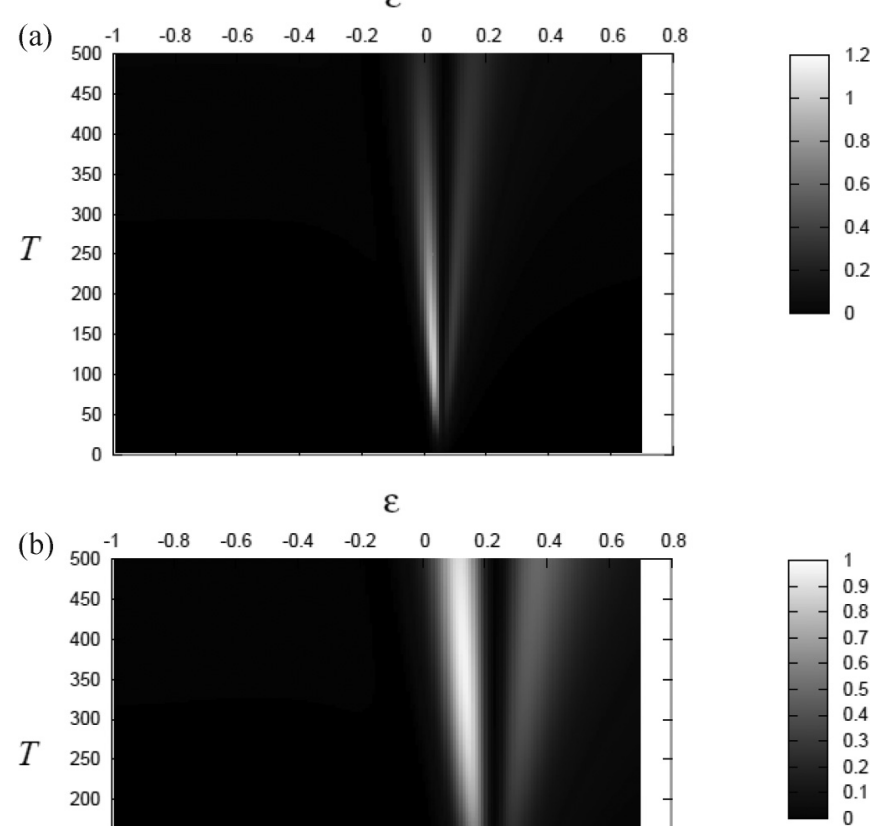

FIG. 9. Figure of merit $Z T$ for model metalloporphyrins dithiolate which have a Fano-like resonance around the Fermi level as a function of temperature $T$ (in $\mathrm{K}$ ) and the level position $\epsilon_{0}$ (in $\mathrm{eV}$ ). The coupling between the $d$ state and the $\pi$ state is (a) 0.2 and (b) $0.4 \mathrm{eV}$. state couples to other molecular states. If the $d$ state couples to HOMO-1, which is a $\sigma$-like molecular orbital, the state produces around the Fermi level a resonance which looks like a Breit-Wigner resonance. Such a peak really comes from a Fano resonance, whose dip is not seen because it is masked by the larger transmission around the Fermi level. If the $d$ state couples, however, to the LUMO orbital, a clear Fano resonance appears around the Fermi level because the dip affects the transmission of the LUMO, which is not completely masked by the transmission of other states.

\section{B. Thermoelectric properties}

From the transmission it is easy to obtain the thermoelectric coefficients by using the equations in Sec. II. We focus especially on the thermopower and figure of merit, which are the most relevant for thermoelectric efficiency. Since we can easily vary the parameters of the model, we study different effects such as the movement of the states across the Fermi level and the change of the coupling between the $d$ state and other molecular states or between the molecule and the leads. We show in Fig. 6 the thermopower and figure of merit calculated at $T=250 \mathrm{~K}$ for Fano and Breit-Wigner-like resonances as a function of the position of the state that gives rise to them. As can be seen, the thermopower shows a peak-dip structure, which is a consequence of the change of the derivative of the transmission as the resonance crosses $E_{\mathrm{F}}$. Such a structure is asymmetric in the first case because
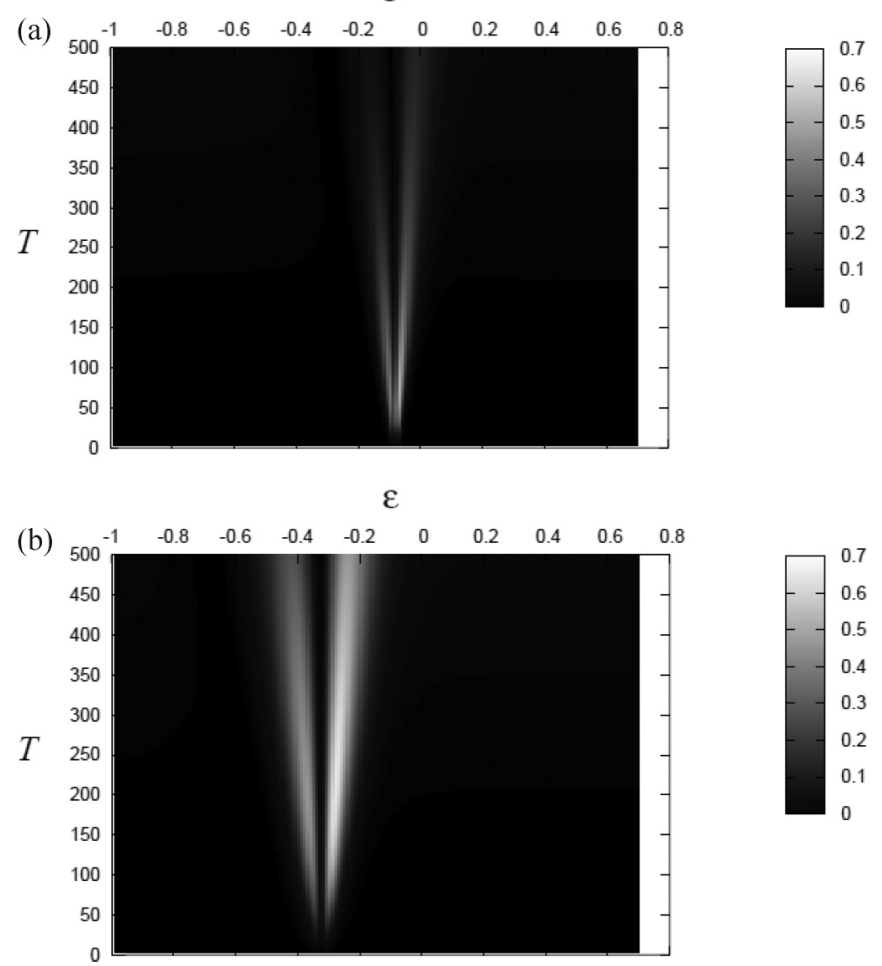

FIG. 10. Figure of merit $Z T$ for model metalloporphyrins dithiolate which have a Breit-Wigner-like resonance around the Fermi level as a function of temperature $T$ (in $\mathrm{K}$ ) and the level position $\epsilon_{0}$ (in $\mathrm{eV}$ ). The coupling between the $d$ state and the $\sigma$ state is (a) 0.4 and (b) $0.8 \mathrm{eV}(\mathrm{b})$. 

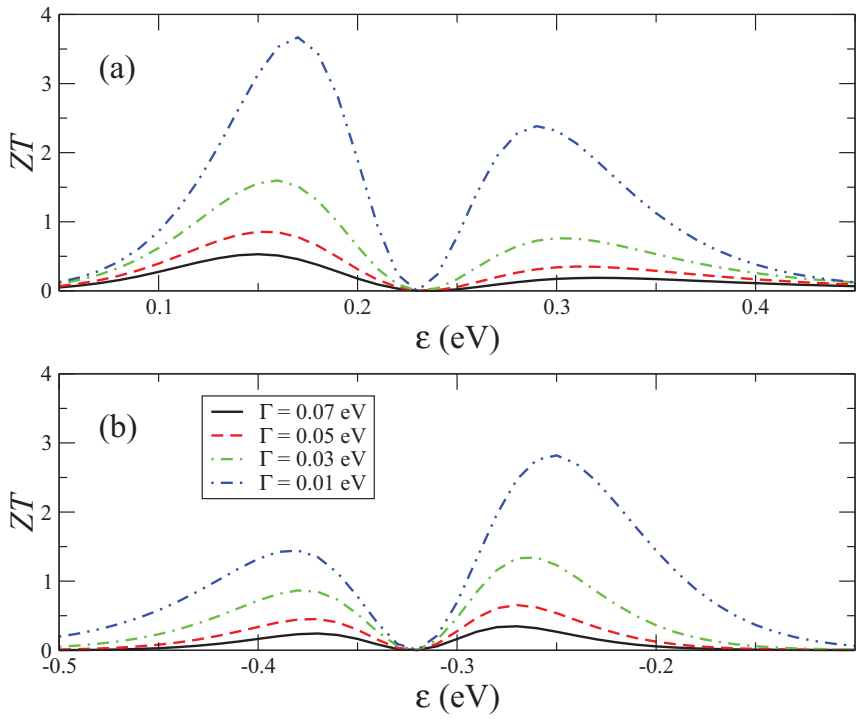

FIG. 11. (Color online) Figure of merit $Z T$ for a model metalloporphyrins dithiolate with (a) a Fano and (b) a Breit-Wigner-like resonance in the HOMO-LUMO gap, calculated at $T=250 \mathrm{~K}$, for various coupling strengths between the HOMO and LUMO and the electrodes. The coupling between the $d$ state and the $\pi$ or $\sigma$ states is (a) 0.4 and (b) $0.8 \mathrm{eV}$.

the Fano resonance is also asymmetric, as it is made of a resonance followed by an antiresonance. In the second case it is also slightly asymmetric because the resonance is not exactly in the middle of the HOMO-LUMO gap. The figure of merit, which reaches values as high as 0.86 , follows roughly the square of the thermopower and therefore has two peaks and a dip, which corresponds to the highest point of each resonance. Notice also both quantities $S$ and $Z T$ are larger in the Fano case due to more pronounced changes in the derivative when the antiresonance follows the resonance.

One effect that can influence the values of the thermopower and figure of merit is the coupling $V$ between the $d$ level and the corresponding molecular level. This can be done, e.g., by using a different metallic atom or straining/compressing the molecule. As can be seen, changing such coupling increases the absolute value of both quantities, especially in the Breit-Wigner-like case, but the change is not very large because the only difference in the transmission is due to an increase of the width of the resonances, which does not affect the derivative too much. This seems to indicate a relative robustness of the absolute value of $S$ and $Z T$ as a function of the metallic atom or small molecular conformation changes. When the coupling increases, there is also a movement of the peaks and dips to lower or higher energies, which is produced by the increase of the separation between levels.

The total evolution of both quantities as a function of the level position and temperature for both couplings is shown in Figs. 7, 8, 9, and 10. As can be seen, the peak-dip structures remain the same for large temperature ranges. Both quantities show high peaks and dips at relatively low and intermediate temperatures, up to $\sim 300 \mathrm{~K}$, and tend to slightly decrease beyond room temperature. According to this, the most efficient heat-to-electricity conversion (large $S$ and ZT) can be achieved in the case Fano resonances at temperatures close to room temperature.

The figure of merit is large but not too large. As previously stated, the value of the figure of merit is capped due to the relatively large transmission at the Fermi level and the presence of the other spin channel. The transmission of both channels can, however, be decreased by reducing the coupling between the molecule and the electrodes, which decreases the width of all transmission resonances and reduces the transmission in the middle. We show in Fig. 11 the figure of merit calculated around the peak-dip structure for various coupling strengths or $\Gamma$ matrices ${ }^{63}$ between the levels and the electrodes. As can be seen, the smaller the coupling or the corresponding $\Gamma$ matrix is, the larger the figure of merit is. For small couplings it can reach values as large as 3.7 . For such small couplings a note of caution should be added, however, since the width of the resonances is so small that strong correlations could change the picture of the physical properties.

\section{CONCLUSIONS}

The thermoelectric properties of junctions with states close to the Fermi level have been calculated using analytical derivations and a simple model. A spin-polarized first-principles calculation of a junction made of a metalloporphyrins dithiolate molecule between gold electrodes has also been included as a realistic example. The Fano and Breit-Wigner-like resonances greatly enhance the thermopower and figure of merit when they cross the Fermi level. The maximum value of these quantities depends on the coupling between the state that gives rise to the resonance and the other molecular states. The bigger the coupling is, the bigger the thermopower and figure of merit are. Their evolution with temperature has also been studied, and it was found that the largest efficiency, corresponding to the largest figure of merit, can be achieved at temperatures close to room temperature. Finally, the coupling between the molecule and the electrodes was also taken into account, and it was found that reducing it greatly enhances the figure of merit, which can reach values larger than 1 .

\section{ACKNOWLEDGMENTS}

The research presented in this paper was funded by the Spanish MICINN through Grant No. FIS2012-34858 and by the Marie Curie network NanoCTM. V.M.G.S. thanks the Spanish Ministerio de Economía y Competitividad for a Ramón y Cajal fellowship (RYC-2010-06053). R.R.F. thanks Consejería de Educación del Principado de Asturias for a Severo Ochoa grant (Grant No. BP11-069).
${ }^{1}$ J. Ferrer and V. M. García-Suárez, J. Mater. Chem. 19, 1696 (2009).

${ }^{2}$ A. Aviram and M. A. Ratner, Chem. Phys. Lett. 52, 9071 (1974).
${ }^{3}$ V. M. García-Suárez and C. J. Lambert, Nanotechnology 19, 455203 (2008). 
${ }^{4}$ P. G. Piva, G. A. DiLabio, J. L. Pitters, J. Zikovsky, M. Rezeq, S. Dogel, W. A. Hofer, and R. A. Wolkow, Nature (London) 435, 658 (2005).

${ }^{5}$ J. He, B. Chen, A. K. Flatt, J. J. Stephenson, C. D. Doyle, and J. M. Tour, Nat. Mater. 5, 63 (2006).

${ }^{6}$ D. P. Long, J. L. Lazorcik, B. A. Mantooth, M. H. Moore, M. A. Ratner, A. Troisi, Y. Yao, J. W. Ciszek, J. M. Tour, and R. Shashidhar, Nat. Mater. 5, 901 (2006).

${ }^{7}$ O. Entin-Wohlman, Y. Imry, and A. Aharony, Phys. Rev. B 82, 115314 (2010).

${ }^{8}$ A. N. Jordan, B. Sothmann, R. Sánchez, and M. Büttiker, Phys. Rev. B 87, 075312 (2013).

${ }^{9}$ Y. Dubi and M. Di Ventra, Rev. Mod. Phys. 83, 131 (2011).

${ }^{10}$ J. Koch, F. von Oppen, Y. Oreg, and E. Sela, Phys. Rev. B 70, 195107 (2004).

${ }^{11}$ K. Baheti, J. A. Malen, P. Doak, P. Reddy, S.-Y. Jang, T. D. Tilley, A. Majumdar, and R. A. Segalman, Nano Lett. 8, 715 (2008).

${ }^{12}$ M. Paulsson and S. Datta, Phys. Rev. B 67, 241403(R) (2003).

${ }^{13}$ A. Tan, S. Sadat, and P. Reddy, Appl. Phys. Lett. 96, 013110 (2010).

${ }^{14}$ X. Zheng, W. Zheng, Y. Wei, Z. Zeng, and J. Wang, J. Chem. Phys. 121, 8537 (2004).

${ }^{15}$ B. Wang, Y. Xing, L. Wan, Y. Wei, and J. Wang, Phys. Rev. B 71, 233406 (2005).

${ }^{16}$ M. Wierzbicki and R. Swirkowicz, J. Phys. Condens. Matter 22, 185302 (2010).

${ }^{17}$ F. Pauly, J. K. Viljas, and J. C. Cuevas, Phys. Rev. B 78, 035315 (2008).

${ }^{18}$ A. Tan, J. Balachandran, S. Sadat, V. Gavini, B. D. Dunietz, S.-Y. Jang, and P. Reddy, J. Am. Chem. Soc. 133, 8838 (2011).

${ }^{19}$ M. Bürkle, L. A. Zotti, J. K. Viljas, D. Vonlanthen, A. Mishchenko, T. Wandlowski, M. Mayor, G. Schön, and F. Pauly, Phys. Rev. B 86, 115304 (2012).

${ }^{20}$ J. Balachandran, P. Reddy, B. D. Dunietz, and V. Gavini, Chem. Phys. Lett. 3, 1962 (2012).

${ }^{21}$ C. M. Finch, V. M. García-Suárez, and C. J. Lambert, Phys. Rev. B 79, 033405 (2009).

${ }^{22}$ Y.-S. Liu and Y.-C. Chen, Appl. Phys. Lett. 98, 213103 (2011).

${ }^{23}$ R. Stadler and T. Markussen, J. Chem. Phys. 135, 154109 (2011).

${ }^{24}$ B. C. Hsu, C.-Y. Lin, Y.-S. Hsieh, and Y.-C. Chen, Appl. Phys. Lett. 101, 243103 (2012).

${ }^{25}$ D. Nozaki, H. Sevincli, W. Li, R. Gutiérrez, and G. Cuniberti, Phys. Rev. B 81, 235406 (2010).

${ }^{26}$ R. Venkatasubramanian, E. Siivola, T. Colpitts, and B. O'Quinn, Nature (London) 413, 597 (2001).

${ }^{27}$ P. Reddy, S.-Y. Jang, R. A. Segalman, and A. Majumdar, Science 315, 1568 (2007)

${ }^{28}$ J. A. Malen, P. Doak, K. Baheti, T. D. Tilley, A. Majumdar, and R. A. Segalman, Nano Lett. 9, 3406 (2009).

${ }^{29}$ J. A. Malen, S. K. Yee, A. Majumdar, and R. A. Segalman, Chem. Phys. Lett. 491, 109 (2010).

${ }^{30}$ S. K. Yee, J. A. Malen, A. Majumdar, and R. A. Segalman, Nano Lett. 11, 4089 (2011).

${ }^{31}$ O. Bubnova, Z. U. Khan, A. Maiti, S. Braun, M. Fahlman, M. Berggren, and X. Crispin, Nat. Mater. 10, 429 (2011).

${ }^{32}$ J. R. Widawsky, P. Darancet, J. B. Neaton, and L. Venkataraman, Nano Lett. 12, 354 (2011).

${ }^{33}$ P. Trocha and J. Barnaś, Phys. Rev. B 85, 085408 (2012).
${ }^{34}$ O. Karlström, H. Linke, G. Karlström, and A. Wacker, Phys. Rev. B 84, 113415 (2011).

${ }^{35}$ P. Murphy, S. Mukerjee, and J. Moore, Phys. Rev. B 78, 161406 (2008).

${ }^{36}$ A. Popescu and P. M. Haney, Phys. Rev. B 86, 155452 (2012).

${ }^{37}$ M. Leijnse, M. R. Wegewijs, and K. Flensberg, Phys. Rev. B 82, 045412 (2010).

${ }^{38}$ V. Kaliginedi, P. Moreno-García, H. Valkenier, W. Hong, V. M. García-Suárez, P. Buiter, J. L. H. Otten, J. C. Hummelen, C. J. Lambert, and T. Wandlowski, J. Am. Chem. Soc. 134, 5262 (2012).

${ }^{39}$ V. V. Maslyuk, S. Achilles, and I. Mertig, Solid State Commun. 150, 505 (2010).

${ }^{40}$ Notice that, in general, in cases with spin polarization and no spin-orbit coupling, the orientation between the spin and the lattice coordinates is arbitrary, and therefore, it is not possible to determine the spin-filtering behavior of the system without coupling to a ferromagnetic material.

${ }^{41}$ N. R. Claughton and C. J. Lambert, Phys. Rev. B 53, 6605 (1996).

${ }^{42}$ C. Rovira, K. Kunc, J. Hutter, P. Ballone, and M. Parrinello, J. Phys. Chem. A 101, 8914 (1997).

${ }^{43}$ M.-S. Liao and S. Scheiner, J. Chem. Phys. 117, 205 (2002).

${ }^{44}$ M. Palummo, C. Hogan, F. Sottile, P. Bagalá, and A. Rubio, J. Chem. Phys. 131, 084102 (2009).

${ }^{45}$ N. Wang, H. Liu, J. Zhao, Y. Cui, Z. Xu, Y. Ye, M. Kiguchi, and K. Murakoshi, J. Phys. Chem. C 113, 7416 (2009).

${ }^{46}$ V. M. García-Suárez, R. Ferradás, D. Carrascal, and J. Ferrer, Phys. Rev. B 87, 235425 (2013).

${ }^{47}$ R. Ferradás, V. M. García-Suárez, and J. Ferrer, J. Phys. Condens. Matter 25, 325501 (2013).

${ }^{48}$ J. Otsuki, Coord. Chem. Rev. 254, 2311 (2010).

${ }^{49}$ G. Sedghi, V. M. Garcí-Suárez, L. J. Esdaile, H. L. Anderson, C. J. Lambert, S. Martín, D. Bethell, S. J. Higgins, M. Elliott, N. Bennett, J. E. Macdonald, and R. J. Nichols, Nat. Nanotechnol. 6, 517 (2011).

${ }^{50}$ W. Kohn and L. J. Sham, Phys. Rev. 140, A1133 (1965).

${ }^{51}$ J. M. Soler, E. Artacho, J. D. Gale, A. García, J. Junquera, P. Ordejón, and D. Sánchez-Portal, J. Phys. Condens. Matter 14, 2745 (2002).

${ }^{52}$ S. G. Louie, S. Froyen, and M. L. Cohen, Phys. Rev. B 26, 1738 (1982).

${ }^{53}$ J. P. Perdew, K. Burke, and M. Ernzerhof, Phys. Rev. Lett. 77, 3865 (1996).

${ }^{54}$ V. I. Anisimov, J. Zaanen, and O. K. Andersen, Phys. Rev. B 44, 943 (1991).

${ }^{55}$ M. S. Liao and S. Scheiner, J. Chem. Phys. 117, 205 (2002).

${ }^{56}$ P. M. Panchmatia, B. Snayal, and P. M. Oppeneer, Chem. Phys. 343, 47 (2008).

${ }^{57}$ A. R. Rocha, V. M. García-Suárez, S. Bailey, C. Lambert, J. Ferrer, and S. Sanvito, Phys. Rev. B 73, 085414 (2006).

${ }^{58}$ J. Ferrer, C. J. Lambert, V. M. García-Suárez, D. Zs. Manrique, D. Visontai, L. Oroszlani, R. Ferradás, I. Grace, K. Guillemot, H. Sadeghi, and L. A. Algharagholy (unpublished).

${ }^{59}$ Notice the value of the figure of merit would be further decreased by taking into account the phonon thermal conductance $\kappa_{p}$, i.e., $Z T=$ $S^{2} G T /\left(\kappa+\kappa_{p}\right)$. In this paper we consider only the electronic contribution. In general, the phonon contribution, which can be obtained by calculating the dynamical matrix, ${ }^{60}$ can be relatively large at high temperatures if perfect electrodes are used. However, it can be greatly decreased by nanostructuring the electrodes to 
include phonon band gaps or using disordered electrodes that increase phonon scattering (Refs. 9, 61, and 62).

${ }^{60}$ T. Markussen, A.-P. Jauho, and M. Brandbyge, Phys. Rev. B 79, 035415 (2009).

${ }^{61}$ G. Fagas, A. G. Kozorezov, C. J. Lambert, J. K. Wigmore, A. Peacock, A. Poelaert, and R. den Hartog, Phys. Rev. B 60, 6459 (1999).

${ }^{62}$ A. Kambili, G. Fagas, V. I. Fal'ko, and C. J. Lambert, Phys. Rev. B 60, 15593 (1999).
${ }^{63}$ As a simplification we assume the coupling of HOMO-1 does not change. This is, in general, not correct since the coupling of all states changes when the molecule gradually uncouples from the leads but will allow us to see just the effect of decreasing the coupling of the HOMO and LUMO. Notice decreasing the HOMO-1 coupling would further enhance the figure of merit since the transmission would be even lower at the Fermi level. This is also the reason why the increase of the figure of merit saturates for very small couplings. 